UCL/HEP 2000-08

OPAL CR444

\title{
A high- $Q^{2}$ measurement of the photon structure function $F_{2}^{\gamma}$ at LEP2
}

\author{
Russell J. Taylor \\ Department of Physics and Astronomy, UCL, \\ Gower Street, London WC1E 6BT, United Kingdom
}

\begin{abstract}
The photon structure function $F_{2}^{\gamma}\left(x, Q^{2}\right)$ has been measured at $\left\langle Q^{2}\right\rangle$ of $706 \mathrm{GeV}^{2}$ using a sample of two-photon events with a scattered electron observed in the OPAL electromagnetic endcap calorimeter. The data were taken during the years 1997-1999, when LEP operated at $\mathrm{e}^{+} \mathrm{e}^{-}$centre-of-mass energies ranging from 183 to $202 \mathrm{GeV}$, and correspond to an integrated luminosity of $424 \mathrm{pb}^{-1}$. This analysis represents the highest $\left\langle Q^{2}\right\rangle$ measurement of $F_{2}^{\gamma}$ made to date.
\end{abstract}

\section{INTRODUCTION}

We present a measurement of the hadronic photon structure function $F_{2}^{\gamma}\left(x, Q^{2}\right)$ at a higher value of the average momentum transfer squared, $\left\langle Q^{2}\right\rangle$, than has ever previously been reported. The measurement of $F_{2}^{\gamma}$ is interesting because of its potential to test perturbative QCD $[1,2]$. In the high- $Q^{2}$ domain the perturbatively calculable point-like contribution to $F_{2}^{\gamma}$, which rises logarithmically with $Q^{2}$, dominates over the non-perturbative hadron-like part.

The structure function $F_{2}^{\gamma}$ has been measured at $\left\langle Q^{2}\right\rangle$ of $706 \mathrm{GeV}^{2}$ using a sample of single-tagged two-photon events recorded by the OPAL detector between 1997 and 1999. These events (also referred to as $\gamma^{\star} \gamma$ events) can be regarded as deep inelastic scattering of an electron on a quasi-real photon, and the flux of quasi-real photons can be calculated using the equivalent photon approximation [3].

To study $F_{2}^{\gamma}\left(x, Q^{2}\right)$ the distribution of events in $x$ and $Q^{2}$ is needed. These variables are related to experimentally measurable quantities by

$$
Q^{2}=2 E_{\mathrm{b}} E_{\mathrm{tag}}\left(1-\cos \theta_{\mathrm{tag}}\right)
$$

and

$$
x=\frac{Q^{2}}{Q^{2}+W^{2}+P^{2}},
$$

where $E_{\mathrm{b}}$ is the energy of the beam electron, $E_{\mathrm{tag}}$ and $\theta_{\mathrm{tag}}$ are the energy and polar angle of the deeply inelastically scattered electron, $W^{2}$ is the invariant mass 
squared of the hadronic final state and $P^{2}=-p^{2}$, where $p$ is the four-momentum of the quasi real target photon. The requirement that the associated electron is not visible in the detector ensures that $P^{2} \ll Q^{2}$, so $P^{2}$ can be neglected when calculating $x$ from Equation 2 .

\section{DATA SELECTION}

This analysis uses data from the 1997 to 1999 LEP runs, with $\mathrm{e}^{+} \mathrm{e}^{-}$centre-ofmass energies ranging from 183 to $202 \mathrm{GeV}$. The total integrated $\mathrm{e}^{+} \mathrm{e}^{-}$luminosity is $424 \mathrm{pb}^{-1}$. Candidate $\gamma^{\star} \gamma \rightarrow$ hadrons events are required to satisfy the following selection criteria, in addition to several technical cuts to ensure good detector status and track quality.

1. A tagged electron is required; that is, a cluster in the OPAL electromagnetic endcap calorimeter with an energy of at least $0.6 E_{\mathrm{b}}$ and a polar angle $\theta$ in the range $230-500 \mathrm{mrad}$ with respect to either beam direction.

2. The energy of the most energetic electromagnetic cluster in the hemisphere opposite to that which contains the tagged electron must be less than $0.25 E_{\mathrm{b}}$.

3. The number of tracks originating from the hadronic final state must be at least 3.

4. The visible invariant mass $W_{\text {vis }}$ of the hadronic system is required to be in the range $2.5 \mathrm{GeV} \leq W_{\text {vis }} \leq 50 \mathrm{GeV}$.

5. The number of objects (tracks plus unassociated clusters), belonging to the hadronic final state must be at least 9 .

6. The energy deposited in a cone of $200 \mathrm{mrad}$ half-angle about the direction of the tag, excluding the tag itself, must not be more than $2 \mathrm{GeV}$.

Cuts 1-4 select a sample of candidate single-tag hadronic two-photon events, with double-tag events excluded by cut 2. Events with leptonic final states are rejected by cuts 3 and 5 . The invariant mass cuts have two functions. The lower limit removes the low- $W$ region which is dominated by resonance production and is very difficult to model accurately. The upper limit rejects background events from hadronic decays of $\mathrm{Z}^{0}$ bosons, as does cut 6 .

A total of 348 events pass these cuts, with the data covering the range $270 \mathrm{GeV}^{2}<$ $Q^{2}<2200 \mathrm{GeV}^{2}$. There is a two sigma difference between the number of events selected in the 1997/8 data and that recorded in 1999, with the 1999 data lying below the Monte Carlo expectation, particularly at low $W_{\text {vis }}$. No explanation has been found for this. The larger samples of $\gamma^{\star} \gamma$ events with the electron tagged in subdetectors at lower polar angles are consistent between the two periods, suggesting that the observed difference could well be purely statistical. However, as a precaution, the difference is included in the systematic error in this preliminary analysis. 
The OPAL LEP1 analysis of $F_{2}^{\gamma}$ using tags in the same subdetector [4] found the trigger efficiency to be $100 \%$. The present analysis uses a tighter set of cuts, thus no inefficiency is to be expected, and a trigger efficiency of $100 \%$ is assumed.

\section{MONTE CARLO MODELLING AND BACKGROUND}

Monte Carlo programs are used to simulate $\gamma^{\star} \gamma$ events and to provide background estimates. The Monte Carlo generator used to simulate signal $\gamma^{\star} \gamma$ multiperipheral events is HERWIG $5.9+k_{\mathrm{t}}(\mathrm{dyn})$ [5]. The GRV LO [6] parameterisation of $F_{2}^{\gamma}$ was used as the input structure function.

The dominant background comes from the reaction $\mathrm{Z}^{0} / \gamma^{\star} \rightarrow$ hadrons. Also significant are non-multiperipheral four-fermion processes with $\mathrm{e}^{+} \mathrm{e}^{-} \mathrm{q} \overline{\mathrm{q}}$ final states and the QED process $\gamma^{\star} \gamma \rightarrow \tau^{+} \tau^{-}$. Less severe sources of background are estimated to account for around $1 \%$ of the data sample.

Figure 1 shows comparisons between data and Monte Carlo distributions. Figure 1(b) shows the polar angle of the tagged electron. It can be seen that the Monte Carlo is somewhat higher than the data in the polar range 260-350 mrad. Turning to variables describing the hadronic final state, it can be seen that the number of charged tracks is reasonably well described, Figure 1(c), but that the Monte Carlo lies above the data at low $W_{\text {vis }}$ in Figure 1(d) - which correlates with high $x$.

\section{DETERMINATION OF $F_{2}^{\gamma}$}

The perennial problem in measurements of $F_{2}^{\gamma}$ is that, because the $\gamma^{\star} \gamma$ centre-ofmass system does not coincide with the laboratory system, the hadronic final state, which must be measured to determine $W$, is only partially observed in the detector. This leads to a dependence of the $F_{2}^{\gamma}$ measurement on the Monte Carlo modelling, which is needed for the unfolding process used to relate the visible distributions to the underlying $x$ distribution.

In the high- $Q^{2}$ measurement presented here, however, the situation is not as serious as at lower values of $Q^{2}$. Because of the larger tagging angle, the hadronic final state has much more transverse momentum and as a consequence is better contained in the detector. Figure 2 shows the correlation between the measured invariant mass $W_{\text {vis }}$ and the generated $W$ as given by HERWIG $5.9+k_{\mathrm{t}}$ (dyn). It can be seen that the correlation is maintained throughout. This is in contrast with the situation observed in the lower $Q^{2}$ analysis [7] where the correlation deteriorates at high $\mathrm{W}$. As a consequence of this the result can be expected to have a smaller dependence on the Monte Carlo modelling of the hadronic final state.

After subtraction of background, the data are unfolded on a linear scale in $x$ in the range $0.1 \leq x \leq 0.98$ using the GURU program [8]. Each data point is corrected for radiative effects and bin-centre corrections are applied. In Figure 3 the data are compared to several theoretical calculations. The leading order 

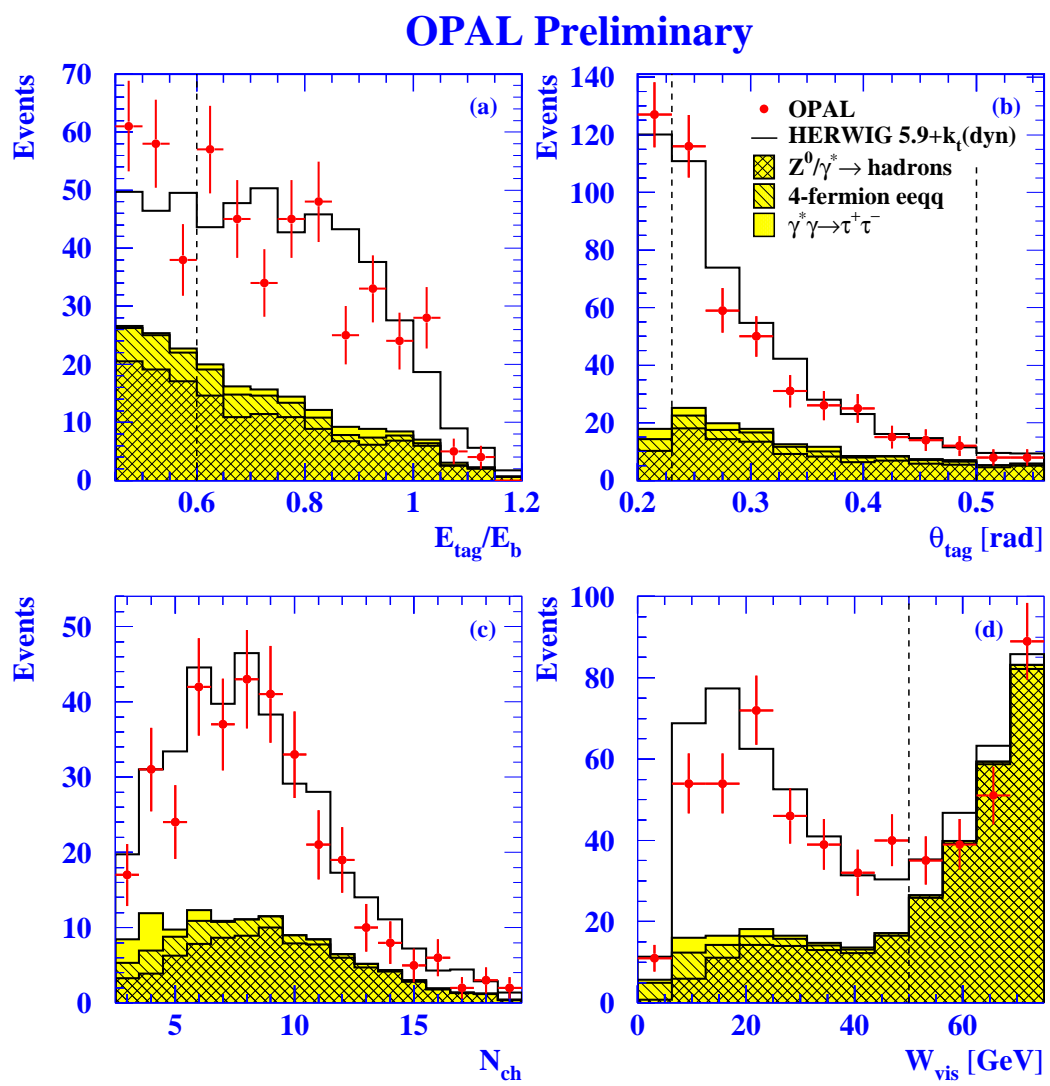

FIGURE 1. Comparison of data distributions with the Monte Carlo prediction. The open histogram is the sum of the HERWIG $5.9+k_{\mathrm{t}}(\mathrm{dyn})$ prediction and the contributions of the major background sources (shown as shaded histograms). All selection cuts have been applied, except for any cut on the variable in the plot (indicated as dashed lines). The distributions shown are: (a) $E_{\mathrm{tag}} / E_{\mathrm{b}}$, the energy of the tagged electron as a fraction of the beam energy, (b) $\theta_{\mathrm{tag}}$, the polar angle of the tagged electron, (c) $N_{\mathrm{ch}}$, the number of tracks originating from the hadronic final state, and (d) $W_{\text {vis }}$, the measured invariant mass of the hadronic final state.

parameterisations of $F_{2}^{\gamma}$ from GRV, SaS1d [9] and WHIT1 [10], which all include a contribution from massive charm quarks, are described in detail in reference [2]. The naive quark-parton model (QPM) simulates only the point-like component of $F_{2}^{\gamma}$, and is calculated for four active flavours with masses of $0.325 \mathrm{GeV}$ for light quarks and $1.5 \mathrm{GeV}$ for charm quarks. It can be seen that in this high- $Q^{2}$ regime the differences between the models are relatively small, particularly in the central $x$-region. The differences between the QPM and the other models are much smaller than at lower $Q^{2}$, where the photon has been shown to have a significant hadronlike component [7]. All the predictions are compatible with the data in three of the $x$ bins, but overshoot the data in one bin. 


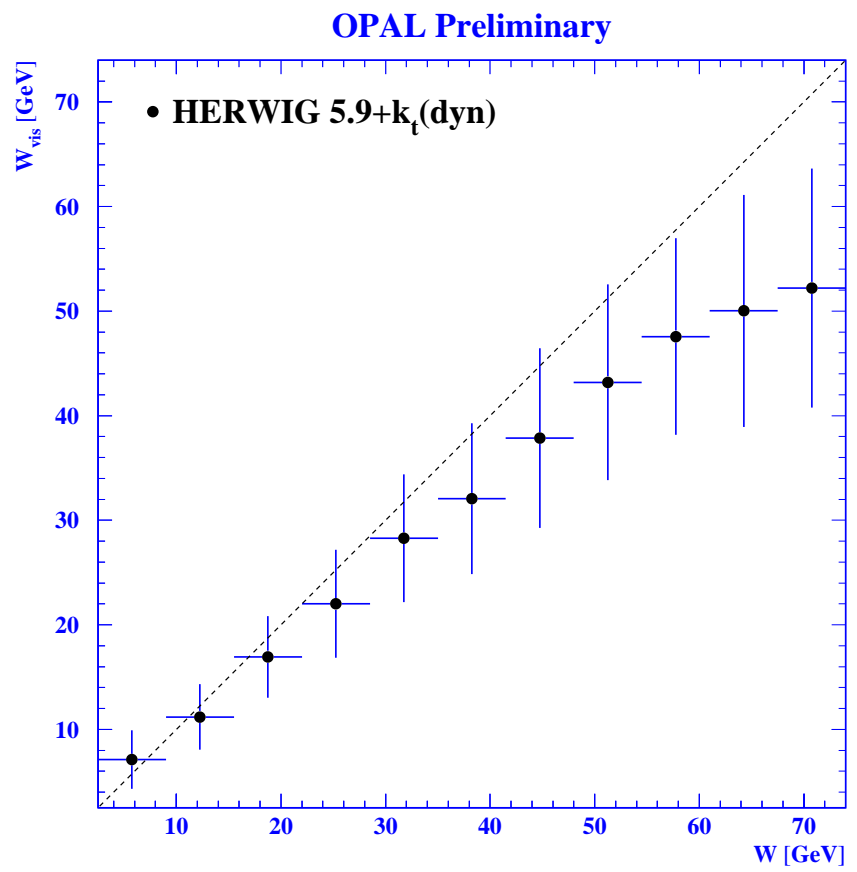

FIGURE 2. The correlation between the generated hadronic invariant mass and the measured value, as given by HERWIG $5.9+k_{\mathrm{t}}(\mathrm{dyn})$. The vertical error bars represent the spread within each bin. The dashed line corresponds to perfect correlation.

\section{CONCLUSIONS}

The photon structure function $F_{2}^{\gamma}$ has been measured using deep inelastic electron-photon scattering events recorded by the OPAL detector during the years 1997-1999. The $\left\langle Q^{2}\right\rangle$ value of $706 \mathrm{GeV}^{2}$ represents the highest measured thus far. $F_{2}^{\gamma}$ has now been measured by OPAL at $\left\langle Q^{2}\right\rangle$ values ranging from $1.9-706 \mathrm{GeV}^{2}$.

\section{REFERENCES}

1. E. Witten, Nucl. Phys. B120 (1977) 189;

A. Cordier and P.M. Zerwas, in ECFA Workshop on LEP200, Vol. 1, CERN 87-08, ECFA 87/108, edited by A. Böhm and W. Hoogland, p. 242.

2. R. Nisius, Phys. Rep. 332 (2000) 165, hep-ex/0006032.

3. C.F. von Weizsäcker, Z. Phys. 88 (1934) 612;

E.J. Williams, Phys. Rev. 45 (1934) 729;

V.M. Budnev et al., Phys. Rep. 15 (1975) 181.

4. OPAL Collaboration, K. Ackerstaff et al., Z. Phys. C74 (1997) 33.

5. G. Marchesini et al., Comp. Phys. Comm. 79 (1992) 465. 


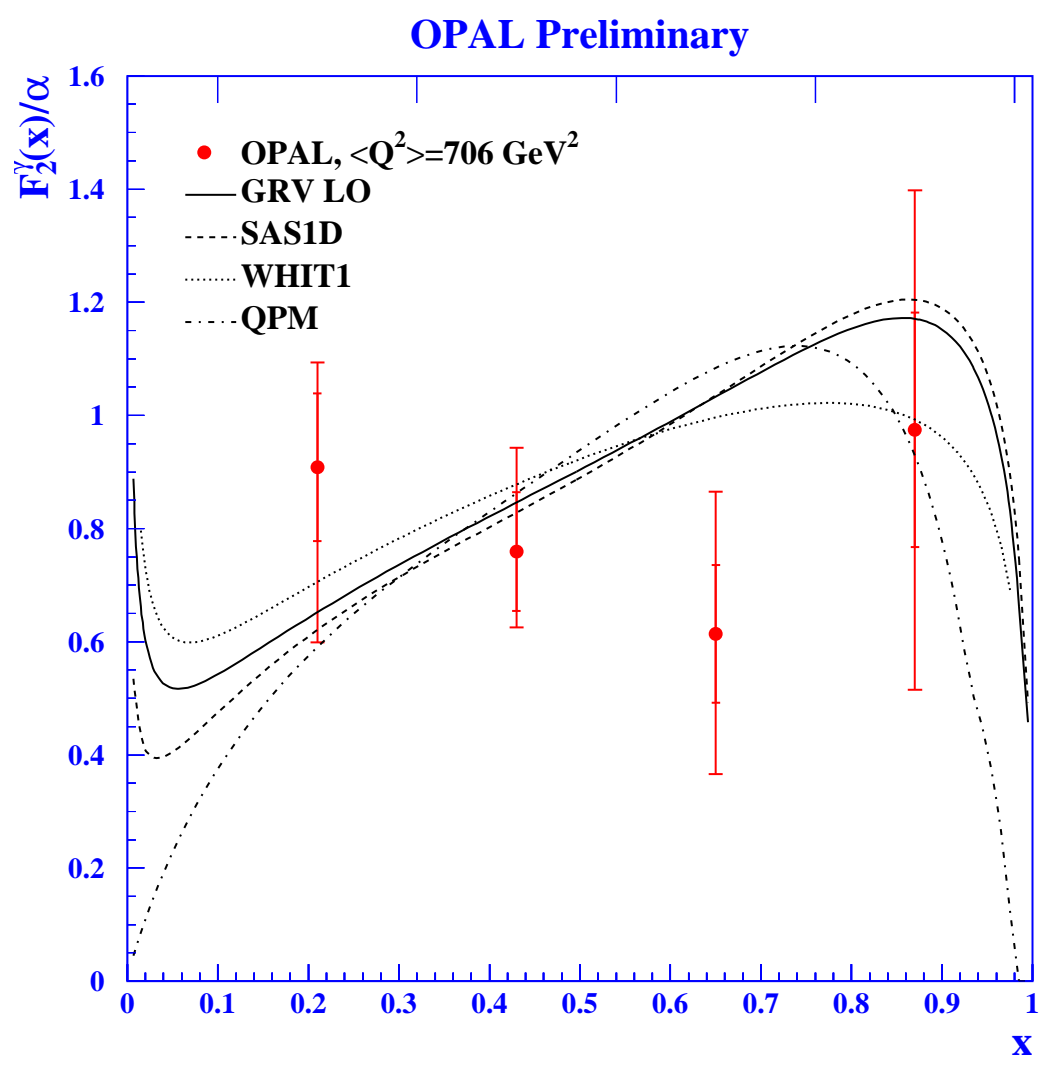

FIGURE 3. The measured $F_{2}^{\gamma}$ at $\left\langle Q^{2}\right\rangle=706 \mathrm{GeV}^{2}$. The inner bars indicate the statistical error and the full bars the total error. The bin boundaries are indicated by the vertical lines at the top of the figure. The curves show the predictions of the GRV LO, SaS1d, WHIT1 and QPM structure functions, all for the $\left\langle Q^{2}\right\rangle$ value of the sample.

The LEP Working Group for Two-Photon Physics, ALEPH, L3 and OPAL Collaborations, Comparison of Deep Inelastic Electron-Photon Scattering Data with the Herwig and Phojet Monte Carlo Models, CERN-EP/2000-109, Submitted to Eur. Phys. J. C.

6. M. Glück, E. Reya and A. Vogt, Phys. Rev. D45 (1992) 3986;

M. Glück, E. Reya and A. Vogt, Phys. Rev. D46 (1992) 1973.

7. E.W. Clay, these proceedings;

OPAL Collaboration, G. Abbiendi et al., Measurement of the low-x behaviour of the photon structure function $F_{2}^{\gamma}$, CERN-EP/2000-082, hep-ex/0007018, Accepted by Eur. Phys. J. C.

8. A. Höcker, V. Kartvelishvili, Nucl. Instr. and Meth. A372 (1996) 469, hep$\mathrm{ph} / 9509307$.

9. G. A. Schuler and T. Sjöstrand, Z. Phys. C68 (1995) 607, hep-ph/9503384.

10. K. Hagiwara et al., Phys. Rev. D51 (1995) 3197, hep-ph/9406252. 